Revue de l'Institut des langues et cultures

d'Europe, Amérique, Afrique, Asie et Australie

$25 \mid 2016$

De la bibliothèque intérieure à la bibliothèque collective : livres et lectures en Espagne (XVI $\mathrm{I}^{\mathrm{e}} \mathrm{XX \textrm {I } ^ { \mathrm { e } }}$ siècles)

\title{
La bibliothèque de travail de Ramón Gómez de la Serna : portrait de l'artiste en collectionneur
}

La biblioteca de trabajo de Ramón Gómez de la Serna: retrato del artista como coleccionista

The Research Library of Ramón Gómez de la Serna: A Portrait of the Artist as a Collectionist

Laurie-Anne Laget

\section{OpenEdition}

Journals

Édition électronique

URL : http://journals.openedition.org/ilcea/3788

DOI : $10.4000 /$ ilcea.3788

ISSN : 2101-0609

Éditeur

UGA Éditions/Université Grenoble Alpes

Édition imprimée

ISBN : 978-2-84310-322-3

ISSN : $1639-6073$

Référence électronique

Laurie-Anne Laget, « La bibliothèque de travail de Ramón Gómez de la Serna : portrait de l'artiste en collectionneur », ILCEA [En ligne], 25 | 2016, mis en ligne le 31 janvier 2016, consulté le 01 mai 2019 URL : http://journals.openedition.org/ilcea/3788 ; DOI : 10.4000/ilcea.3788

Ce document a été généré automatiquement le 1 mai 2019.

(C) ILCEA 


\title{
La bibliothèque de travail de Ramón Gómez de la Serna : portrait de l'artiste en collectionneur
}

\author{
La biblioteca de trabajo de Ramón Gómez de la Serna: retrato del artista como \\ coleccionista \\ The Research Library of Ramón Gómez de la Serna: A Portrait of the Artist as a \\ Collectionist
}

Laurie-Anne Laget

El que no sabe ya dónde colocar más libros espera
que se invente la estantería imantada que logre
retenerlos en el techo.
Ramón GóMEZ DE LA SERNA, Total de greguerías.

1 Au printemps 1967, Luisa Sofovich, romancière argentine et veuve de Ramón Gómez de la Serna, céda à l'université de Pittsburgh un fonds d'archives composé de livres, de manuscrits inédits (notes, brouillons ou œuvres en préparation) et d'extraits de la correspondance de l'écrivain madrilène disparu en janvier 1963. L'un des artisans de cette acquisition, le professeur Rodolfo Cardona, également auteur de la première monographie critique sur l'œuvre ramonien (1957), a évoqué le processus de transfert et de réception de ce fonds dans un article publié vingt ans plus tard, où il rappelait l'intérêt capital de ce "valiosísimo "laboratorio" para estudiar el procedimiento creativo de un autor" (Cardona, 1989: 15). À juste titre, Luisa Sofovich parlait d'un «trésor ramonien » (id. : 11) : les milliers de fiches jaunes griffonnées à l'encre rouge, la calligraphie redoutable, le chaos des brouillons lacunaires, mais aussi la somme impressionnante des notes prises au fil de la plume, les sujets de prédilection que l'on y voit se dessiner peu à peu et tous les projets esquissés pour de futures œuvres constituent un observatoire privilégié de l'univers créateur de l'écrivain madrilène. C'est un aperçu des coulisses de la création littéraire à travers l'éclairage d'une bibliothèque de travail que nous offrent les archives de Pittsburgh. 
2 Il est vrai qu'on ne pourra ici parler de bibliothèque au sens strict, dans la mesure où seules les œuvres personnelles de l'auteur furent transférées aux États-Unis, notamment les premières éditions de ses livres des années dix et vingt, qui étaient alors quasiment introuvables. Le fonds de Pittsburgh recèle néanmoins une autre forme de bibliothèque, toute ramonienne celle-là: un ensemble de coupures de presse, de documents iconographiques et de fiches manuscrites sur lesquelles l'auteur collait ou recopiait des citations trouvées dans la presse littéraire de l'époque. Il s'agit bien à mon sens d'une bibliothèque ou, à tout le moins, de la "version abrégée d'une bibliothèque ", pour reprendre l'expression de Jennifer Allen à propos de la « vaste collection de citations, qui semble avoir remplacé la collection de livres » que constitue le projet sur les Passages parisiens de Walter Benjamin ([1931]: 12-13). Tout comme l'ouvrage inachevé de Benjamin, les archives de Pittsburgh témoignent, en effet, de la démarche de choix et de recueil des sources adoptée par Ramón pour alimenter ses œuvres futures. Par ailleurs, le fait que ces fragments de bibliothèque soient extraits de la presse est parfaitement cohérent avec la formation littéraire de l'auteur, qui a été modelée par le média journalistique depuis les années dix (Laget, 2012).

On trouve ainsi dans ce fonds des coupures de presse annotées, des portraits ou photographies d'auteurs classiques et modernes, ainsi que plusieurs centaines de feuillets de citations manuscrites. Parmi ce dernier ensemble, certaines citations particulièrement marquantes aux yeux de l'écrivain ont été recopiées plusieurs fois, voire réécrites ou personnalisées. En ce sens, il me semble que l'on peut considérer cette partie des archives ramoniennes comme une forme de matérialisation de la «bibliothèque intérieure » de l'auteur, suivant la définition qu'en donne Pierre Bayard en tant qu' " ensemble de livres [...] sur lequel toute personnalité se construit et qui organise ensuite son rapport aux textes et aux autres » $(2007: 74)$. Pierre Bayard parle également de « livres marquants » et les documents de travail présents dans la bibliothèque de Pittsburgh témoignent bien de l'intérêt de Ramón pour la vie et les textes de certains écrivains qui imprégnèrent son œuvre. En somme, je m'intéresserai ici à la «bibliothèque comme dispositif », pour reprendre l'expression de Stéphane Lojkine (2007), c'est-à-dire comme espace de réflexion et d'imagination autour du livre, un espace «li[é] à un ensemble de pratiques du savoir, et non au seul livre »(Melot, 2007). Il s'agit d'une bibliothèque atypique, faite d'extraits et de fragments choisis par Ramón (le mot selección apparaît souvent comme entête des fiches manuscrites de l'auteur), qui nous renseigne sur la démarche créatrice de l'auteur.

Plus précisément, je me centrerai ici sur les documents de la bibliothèque de travail ramonienne en lien avec les greguerías - la clef de voûte de son œuvre -, à savoir : les recherches menées dans la presse de l'époque (études et recensions critiques d'œuvres d'auteurs étrangers) et les fiches de citations d'auteurs que Ramón recueille sous le titre Gregues ("greguerías pour les intimes», comme il aimait à les appeler). Je voudrais ainsi donner un aperçu de l'espace de travail ramonien, en y analysant les traces que laisse la création littéraire, afin d'offrir une vue d'ensemble sur le contexte dans lequel s'inscrivaient et s'écrivaient les greguerías.

\section{La bibliothèque de travail comme espace de création}

5 La bibliothèque aujourd'hui conservée à la Hillman Library fut un espace de création vivant jusqu'au début des années soixante ${ }^{1}$, ainsi qu'en témoigne une lettre que l'écrivain 
adressa à Rodolfo Cardona en 1958, dans laquelle il évoquait son temps bien occupé par " los artículos y la media semana contemplativa para mis 45 greguerías semanales" (Cardona, 1989: 19). Ce qualificatif - la semaine "contemplative» de création - me semble particulièrement intéressant, car il souligne l'importance des sources d'inspiration pour l'auteur des greguerías. Variation ludique et imagée de l'aphorisme, la greguería repose, en effet, sur un tour de force stylistique : celui de créer une sensation de surprise mâtinée d'évidence poétique, tout en parvenant à susciter l'adhésion du lecteur et à piquer sa curiosité. On peut ainsi lire, parmi les dernières greguerías inédites conservées à Pittsburgh, cette définition gourmande du livre: «Libro: hojaldre de ideas » ou ces deux lectures figurées qui jouent sur les mots à propos de la bibliothèque : «Placard: biblioteca para la ropa »; «El erudito se hace la casa con ladrillos de libros» (Ramón Gómez de la Serna Papers: II. 1.5). On devine l'enjeu des stimulations conceptuelles, visuelles ou sonores pour écrire chaque semaine de nouvelles greguerías (Ramón vivait alors de sa plume grâce à elles). À la différence de l'érudit qui construit sa maison en briques - livres pesants et inertes -, Ramón remplissait sa bibliothèque de citations et de fragments légers, qui l'inspiraient.

Parmi ces sources d'inspiration textuelles, la série de documents la plus remarquable est l'ensemble de 885 feuillets sur lesquels Ramón a recopié des fragments en prose, aphorismes, haïkus et autres extraits de poèmes. On y reconnaît différentes formes habituelles de greguerías, comme le jeu idéogrammatique sur les lettres de l'alphabet, dont Ramón est, on le sait, un expert récidiviste ${ }^{2}$. On peut ainsi lire sur plusieurs fiches ces citations de Victor Hugo : "La G es el cuerno de caza », «L: la pierna con el pie », «Z: el relámpago de Dios ${ }^{3}$ " (Ramón Gómez de la Serna Papers: II. 4.11, passim). On s'aperçoit également qu'au-dessous de cette dernière phrase, Ramón a copié une variante de Leo Longanesi : « La Z tiene una sola ambición: revistar a las otras letras », donnant ainsi à penser qu'il collige sur ces fiches du matériel de recherche, en quête d'idées et de formulations nouvelles pour ses propres textes. L'autre type de greguería présent dans la collection de citations ramonienne est, plus généralement, celui des redéfinitions poétiques. Pour n'en citer qu'un exemple, je retiendrai cette lecture figurée des paupières de Malcolm de Chazal, réécrite (approximativement) par Ramón: "Las pestañas / Son / El candelero arandel / De la mirada » (id. : II. 4. 10). La parenté avec les greguerías est évidente - que l'on songe à cette greguería qui dit que «El monóculo es el llavero de las miradas» (1962 : 223) -, de sorte que l'on peut imaginer que la collection de citations ramonienne permettait à l'auteur de stimuler son imagination greguerística.

7 C'est ce que suggèrent, à tout le moins, les indications que porte Gómez de la Serna en marge des citations qu'il recopie. Outre les appréciations simples - comme le jugement « buena » apposé à une greguería de Malcolm de Chazal, « El perro / Ladra / A la luna / Porque cree / Que es un hueso ${ }^{4} »-$, on trouve parfois consignées des gloses ou des explications de certaines citations. Il en va ainsi pour le néologisme suivant, que Ramón décompose pour expliciter l'image de l'enchinarrado :

Enchinarrado de estrellas (Unamuno).

chinas $=$ piedrecitas .

chinitas $=$ me tiraba chinitas,

Enchinarrado

> convertido en greguería. (Ramón Gómez de la Serna Papers : II. 1. 4. 10, fo 40)

8 Ramón nous livre dans cette note une observation des mécanismes de création de cette image d'Unamuno qui semblent avoir attiré son attention. L'expressivité de la métaphore concentrée autour du néologisme lui vaut de figurer au sein de la collection de citations 
de la bibliothèque de travail ramonienne. Sur ce même modèle, on trouve d'ailleurs dans les manuscrits de l'époque, des greguerías qui jouent sur le néologisme pour créer un effet poétique extrêmement synthétique. Ainsi, "Noche lunilunada» joue sur l'image de la pleine lune et la lumière qu'elle projette ou, au contraire, « paisaje lunigrisado = aquel al que ilumina la luna a través de una gasa de nubes » (Ramón Gómez de la Serna Papers: II. 5. 38. 2, fo 108). On pourrait également citer ce mot-valise par lequel Ramón invente, à partir de cornifero (en réalité, cornígero), "que tiene cuernos ", l'animal cornifiero «que tiene terribles cuernos» (II. 5.37.6, fo 123a). Dans tous ces exemples, on remarque le besoin d'explicitation de Ramón qui est toujours soucieux d'offrir à ses lecteurs des images inédites, mais jamais gratuites pour autant, ni incompréhensibles. C'est déjà ce qu'indiquait la glose du néologisme d'Unamuno: Ramón y révélait la motivation de l'image de l'enchinarrado et lui conférait, dès lors, le statut de greguería.

Comme on pouvait s'y attendre, la bibliothèque de travail donne des indices sur la démarche créatrice de l'écrivain à travers ses travaux de recherche : en l'occurrence, elle fait état de la lecture régulière et minutieuse de diverses proses brèves qui viennent alimenter la poétique du fragment des greguerías. Mais elle nous livre également une partie des sources bibliographiques de l'écrivain.

Un premier outil essentiel en ce sens est l'édition argentine des Selecciones del Reader's Digest et, tout particulièrement, les pages des rubriques de vocabulaire et de citations, que Ramón découpait et collait au dos de certaines fiches de Gregues. Le fonds de Pittsburgh témoigne ainsi du fait que la presse est, dans les années cinquante encore, une source privilégiée de l'auteur, d'autant plus pertinente dans ce cas précis que le magazine Selecciones offre à l'auteur un répertoire de (jeux de) mots et d'images, susceptibles de devenir ferments de greguerías. Parfois, Ramón se contente de souligner un mot: patizambo, huésped, propileo, etc. (Ramón Gómez de la Serna Papers : II. 1. 3. 18, passim). Mais il collectionne aussi des images, comme "Los guijarros entre el agua brillante / tienen la claridad de la perla sobre la blanca garganta » (II. 1. 4, fo 8 ), dont il retient la fin : «Perlas sobre la blanca garganta ». On peut alors trouver un écho - assurément plus trivial - à l'image des perles blanches sur une gorge dans cette greguería : « Collar de perlas: dentadura para las gargantas » (1962: 100). Certes, de même que pour tous les exemples cités jusqu'ici, il est impossible d'affirmer que ces textes mis en évidence par Ramón constituent une source directe de son œuvre. Néanmoins, les fiches ramoniennes nous renseignent sur les recherches menées par l'auteur, afin d'aiguiser son imagination à la lecture de mots inconnus ou jugés particulièrement expressifs et de textes qu'il considérait comme des "greguerizaciones eventuales» d'autres écrivains (1962: 40). C'est un travail de chercheur-collectionneur indispensable pour l'écrivain greguerista qui se donne à voir dans ces citations et documents patiemment colligés par l'auteur.

11 En ce sens, il me semble intéressant de rapprocher ces brouillons et notes préparatoires aux Greguerías d'un dossier laissé de côté dans les inventaires, mais qui en constitue une source complémentaire : il s'agit d'un ensemble important de 75 chemises de coupures de presse de journaux et de revues littéraires, contenant des biographies d'écrivains étrangers (pour la plupart anglo-saxons ou francophones et, dans une moindre mesure, allemands, italiens ou russes), de nombreuses représentations iconographiques de ces auteurs, ainsi que des recensions critiques de leurs œuvres. Dans l'inventaire le plus récent du fonds de Pittsburgh, Brigitta Arden interprète ces matériaux comme une compilation de «clipplings for inspiration, personal interest, or project-related work» (Ramón Gómez de la Serna Papers: IV.40-45). On ne peut malheureusement que formuler des 
hypothèses sur l'usage que pouvait faire Ramón de ces archives, étant donné qu'on en ignore le classement précis : l'actuel inventaire distingue les articles par aire culturelle (littératures nord-américaine et britannique, française, italienne, etc.) et seuls certains dossiers originaux ont été conservés (plusieurs séries d'articles consacrés à Marc Twain, à Charles Dickens, à H. G. Wells, à Francis Jammes, à André Gide, à François Mauriac, à Léon-Paul Fargue, à Honoré de Balzac, à Ada Negri ou à Boccace), peut-être comme étude préparatoire pour de nouveaux portraits littéraires, dans la lignée de la série de Retratos contemporáneos (1941), mais pas uniquement. On regrette surtout aujourd'hui que ces documents ne soient quasiment pas annotés. Le plus souvent, Ramón se contente d'indiquer le nom de l'auteur sur lequel porte l'article.

Il existe tout de même quelques gloses manuscrites plus significatives. Je n'en citerai ici qu'un seul exemple, car il a trait à la greguería. Il s'agit d'un article à propos de la figure de Minou Drouet, prodige hautement controversé de la poésie française des années cinquante, en raison de son jeune âge (elle a alors huit ans) et des mises en question de son autorité littéraire. En reprenant la collection des fiches de Gregues, on s'aperçoit que Ramón y a recopié sur plusieurs fiches quatre des six citations reproduites dans l'article, comme il le signale sur la coupure de presse par une note manuscrite dans laquelle il indique : «copiadas las greguerías. 1955 » (Ramón Gómez de la Serna Papers : IV. 42. 14, fo 9). L'assimilation des extraits de poèmes de Minou Drouet aux greguerías est en soi intéressante, puisqu'elle suggère qu'aux yeux de Ramón ces textes, tout comme ceux de l'ensemble des auteurs réunis dans les fiches de Gregues, font partie d'un même patrimoine/tradition greguerísticos ${ }^{5}$. Pour autant, la découverte de la jeune poète en 1955, alors que Ramón publie cette année-là la première édition de son Total de greguerías (somme quasi définitive de ses " petites phrases »), ne saurait être considérée comme une simple source d'inspiration.

\section{La fonction sociale de la bibliothèque de travail}

13 C'est le prologue du Total de greguerías qui nous fournit alors une nouvelle clef de lecture pour la collection ramonienne d'articles et de citations: «Al mismo tiempo yo recojo en los libros viejos y actuales, así como en las revistas, Greguerías que están bien » (1962: 73). Outre l'identification de possibles sources d'inspiration, les fiches de Gregues offrent une confirmation de la valeur littéraire des greguerías ramoniennes par l'émulation qu'elles ont suscitée. Ramón évoque dans son prologue ce phénomène de "polinación [que] produce sementera y constantemente me llegan "greguerías" de nuevos jóvenes y hasta se publica algún libro bajo su título » $(i d$.$) . Dès l'édition de 1940, en réalité, un chapitre du prologue, le$ numéro VII, est consacré à l'influence des greguerías sur la littérature de leur temps : il débordera bientôt sur le chapitre suivant pour s'étendre sur dix-huit pages dans la seconde édition du Total de greguerías parue en 1962 - la dernière ayant vu le jour du vivant de l'auteur. Si Minou Drouet ne figure pas dans cette longue «compilation » de jeunes auteurs de greguerías inspirés par Ramón (id. : 80) ${ }^{6}$, on y rencontre d'autres noms, comme ceux de Noel Clarasó, Enrique Jardiel Poncela, Tomás Seral y Casas ou Francisco Soler y Pérez et même Federico García Lorca, qui reviennent à plus de 25 reprises dans les fiches de Gregues. L'inventaire de ce corpus (produit en annexe) fait ressortir deux grands types d'auteurs: les autorités - et possibles sources d'inspiration originelles ${ }^{7}-$ comme $^{2}$ Jules Renard, Paul Éluard, Oscar Wilde ou encore Arthur Rimbaud et les épigones déjà cités, parmi lesquels le poète Federico García Lorca, dont j’ai pu recenser pas moins de 
89 occurrences. On remarquera que, si l'essentiel de ces figures de continuateurs de la greguería sont des écrivains espagnols ou latino-américains, Ramón est généreux dans la projection de son ascendant greguerístico et inclut dans sa collection de Gregues nombre d'auteurs et d'artistes contemporains, aussi divers que Jean Cocteau ou Alfred Hitchcock. Il indique aussi, en marge des noms précis, des citations par nationalité (arabe, brésilienne, chinoise, japonaise ou russe) pour dire l'universalité de la greguería ${ }^{8}$.

On touche ici à une nouvelle fonction de la bibliothèque de travail, qui cesse d'être uniquement l'espace privé de la créativité de l'auteur, pour revêtir une fonction sociale en devenant le lieu de construction d'un discours d'auto-promotion autour de la greguería. L'hypothèse que je formule ici est que l'ensemble des fiches étiquetées Gregues constituent le travail préparatoire de défense et illustration de la greguería pour les prologues de futures anthologies de Greguerías.

Il faut alors rappeler l'enjeu rhétorique des prologues ramoniens. Le ton est donné dès les premières pages (rédigées dans les années vingt) : c'est le je de l'écrivain qui s'impose (" Desde 1910 [...] me dedico a la Greguería... »), conforté par diverses tournures impératives (« Hay que dar la breve periodicidad de la vida... » [1962: 21 ; je souligne]). La structure du prologue vise ensuite à renforcer cette affirmation liminaire de l'autorité littéraire ramonienne : par le rappel des polémiques qui entourèrent leur première publication (en raison de leur «atrevimiento », rétorque Ramón [id.:25]); par la revendication du choix de ce titre de Greguería, lequel consacre un genre littéraire qui, avant que Ramón le nomme, n'existait pas ; par la définition en creux de la greguería (qui n'est ni une simple figure rhétorique, ni une devinette ou un bon mot et encore moins un aphorisme) ; par la révélation d'une tradition littéraire ancienne mise à jour par Ramón Gómez de la Serna (une sorte d'histoire du fragment poétique que la greguería vient parachever en lui donnant toute son autonomie); enfin, par le «chemin parcouru » par les greguerías à travers les traductions étrangères et la floraison de variantes dans toute l'Amérique latine (sous la forme de concours littéraires ou de rubriques inspirées de la greguería dans la presse). La nouveauté des années quarante et cinquante est le passage en revue systématique des émules de la greguería: il s'agit désormais, pour l'auteur, de montrer que la greguería est pleinement devenue un genre littéraire à succès $-\mathrm{y}$ compris économique, puisque, Ramón le souligne, "Por lo pronto, la Greguería es como un pequeño valor que circula en Bolsa » (1962: 68). C'est dans ce cadre que l'auteur propose sa sélection personnelle des imitations de greguerías "dignas de citarse » (id.: 62), qu'il est, bien évidemment, le seul à pouvoir reconnaître et distinguer.

L'inventaire des écrivains cités dans la collection de Gregues me semble prendre tout son sens dans cette perspective : Ramón établit ainsi une généalogie de la greguería en tant que genre littéraire légitime et prestigieux pour donner l'impression que sa place au sein de l'histoire littéraire s'enracine dans des fondations antiques (Euripide, Horace ou Ovide), égale les grands noms de la littérature du Siècle d'or (Góngora, Quevedo et Gracián), tout en s'inscrivant dans la modernité de la littérature des avant-gardes (Breton et les surréalistes, García Lorca, etc.). En aval, la liste des continuateurs s'allonge et comprend un nombre croissant d'auteurs prestigieux de l'époque, comme Cernuda ou Neruda. À chaque auteur mentionné correspond une citation-greguería qui vient étayer la démonstration ramonienne et, plus encore, la valider par un argument d'autorité : " Shakespeare gregueriza cuando dice antes que nadie: "El ave del alba", y cuando dice "Los ojos son los locos del corazón". Pascal cuando dice... » (1962 : 39) L'affirmation est présentée comme indiscutable pour les auteurs classiques: Ramón dote la greguería d'une tradition 
littéraire qu'il crée de toutes pièces par l'accumulation de citations ponctuelles. Les auteurs contemporains, eux, font l'objet d'un jugement : Ramón distingue les greguerías " de estupendo cuño » ou simplement «acertadas » de celles qui, au contraire, sont à peine " aceptables », voire " equivocadas » (id. : 63-65). Cette fois-ci, la formulation explicite d'un choix, en plus de la citation-exemple, permet d'asseoir l'autorité ramonienne. Le résultat est une moisson de greguerías (Ramón s'enorgueillit tout particulièrement de ses " suntuosas cosechas de la nueva especie » [id.: 58]) sous la forme d'un florilège de morceaux choisis par l'auteur.

17 Or, toutes ces citations brandies dans le prologue du Total de greguerías proviennent de la collection des Gregues, qui en constitue bien l'étude préparatoire. L'index des auteurs cités dans ces fiches devrait à l'avenir permettre de mieux comprendre, je l'espère, le système de références dans lequel Ramón inscrivait la greguería au moment de la présenter à son public.

\section{Du choix des « ingrédients » : la bibliothèque de travail comme exercice de la collection}

Au-delà de la fonction et des usages que pouvait offrir à Ramón son atypique bibliothèque de travail, il me semble important de rappeler que ces documents étaient jusqu'au début des années soixante indissociables de l'espace du bureau de Gómez de la Serna à Buenos Aires, où ils prenaient place 9 . Si je compare ici ces deux ensembles, c'est parce que je les crois susceptibles d'apporter un éclairage sur la logique qui a présidé à leur constitution et agencement, tout autant que sur les pratiques créatrices de l'auteur.

C'est à dessein que j'ai employé jusqu'ici le terme de collection pour désigner la bibliothèque de travail ramonienne. Car il s'agit là d'un procédé habituel chez cet écrivain, attesté depuis les années vingt, précisément dans l'espace singulier du Torreón ${ }^{10}$. Tout d'abord décrit dans la presse et les milieux littéraires par les témoignages des amis de l'auteur, ce bureau - ou "chambre de travail ${ }^{11}$ ", comme aimait à le désigner Ramón (1924: 679) - fut révélé au public par un premier reportage graphique en 1930. Les lecteurs purent ainsi découvrir en une du quotidien $A B C$ les photographies d'Alfonso ${ }^{12}$ représentant Ramón dans un espace regorgeant d'objets de toutes sortes (un lampadaire, un mannequin en cire grandeur nature ou encore des boules de verre suspendues au plafond) et dont les murs et le plafond étaient recouverts d'images superposées. Chineur bien connu des brocanteurs du marché aux puces de Madrid, le Rastro, Ramón a toujours reconnu qu'il était un incorrigible collectionneur. Du Rastro, il rapportait les objets les plus surprenants auxquels il disait donner une seconde vie en les exposant chez lui. Dans le chapitre qu'il consacre à son bureau dans ses mémoires, l'auteur explique que cette mise en scène spectaculaire lui permettait de « ca[zar] ideas y espe[rar] inspiración » (Gómez de la Serna, 1948 : 494).

À mon sens, il n'est pas anodin que la collection de notes et de fiches qui compose la bibliothèque de travail ramonienne ait pris place au sein de cet espace créatif singulier, car elle obéit à un fonctionnement comparable à celui du bureau. Sans arriver tout à fait à égaler le caractère spectaculaire de ce dernier, les notes manuscrites, articles découpés dans la presse et autres citations diverses ressortissent au même principe de collection et nous donnent à voir l'écrivain en chercheur-chineur, en train de se construire un espace mental et imaginaire bien à lui. Il s'agit, en somme, de deux bibliothèques parallèles : 
l'une visuelle/graphique et l'autre textuelle. De même que les objets collectionnés revêtaient un sens nouveau dans le cadre du bureau ramonien - le mannequin de cire, par exemple, y devenait muse et compagne idéale (car muette...) -, on a vu que les citations recueillies par Ramón étaient autant de pièces qui configuraient peu à peu une généalogie littéraire de la greguería.

Par ailleurs, tant pour la disposition spatiale du bureau que pour les manuscrits, le sens de la collection ramonienne est dans l'effet de surprise que produit la juxtaposition d'objets et d'images disparates et dans le hasard des rencontres parfois heureuses ou suggestives :

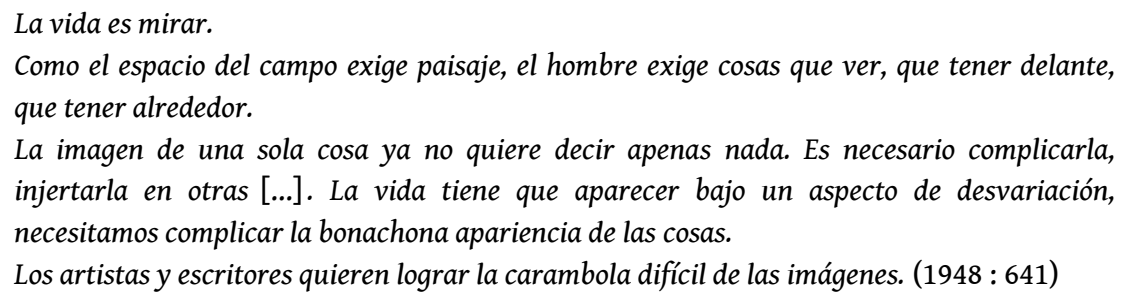

La logique qui préside à cet assemblage "monstrueux », dans le sens étymologique du terme (id. : 494), est celle de l'expérimentation et de la découverte. Le bureau ramonien, saturé de stimulations visuelles, ouvre sur un espace d'imagination, dans lequel la surface des choses s'efface pour laisser affleurer les «rapports insoupçonnés entre les choses » $(1936: 64)$ : le réel ainsi observé devient un champ des possibles, qui promet au spectateur-écrivain une perception renouvelée de la réalité.

On pourrait alors rejoindre la lecture de Walter Benjamin - grand collectionneur, lui aussi, d'objets (jouets d'enfants), de livres et de citations -, qui envisage la pratique de la collection comme un acte de renouvellement du monde. La citation de Je déballe ma bibliothèque est aujourd'hui célèbre. Comparant le collectionneur à un enfant, Benjamin y affirme que,

[...] pour le vrai collectionneur, l'acquisition d'un livre ancien équivaut à sa renaissance. Et en cela réside l'aspect enfant [du collectionneur] [...]. En effet, les enfants commandent au renouvellement des livres comme à une praxis démultipliée, jamais à court. Chez les enfants, l'acte de collectionner n'est qu'un procédé de renouvellement parmi d'autres, tels la peinture des objets, ou le découpage, ou encore le décalque, et, de la sorte, toute la gamme des modes d'acquisition enfantine, depuis la prise en main jusqu'à la nomination, ce sommet. Renouveler le monde - c'est là l'instinct le plus profond dans le désir qu'éprouve le collectionneur d'acquérir de nouveaux objets. ([1931] : 44)

24 À l'instar de celui qui nomme le réel, le collectionneur en produit sa propre version cohérente, qu'il agence et ré-agence constamment. Il s'agit d'un processus dynamique et c'est le cas également chez Ramón Gómez de la Serna, qui ne cesse de compléter la collection-album de son estampario, en y superposant de nouvelles images.

Or, il s'agit bien là d'un "mode d'acquisition »: d'après Benjamin, la pratique de la collection constitue une interprétation du monde, une sorte d'acte démiurgique. Ramón écrit que « se forma un universo » $(1948: 646)$ et évoque l'effet de sa collection d'images en termes de connaissance et d'appréhension des choses:

Puedo decir que si en mi habitación entrase un murciélago se volvería loco o sabio.

El procedimiento para crear este cauchemar de imágenes es obra de la inspiración y del delirio [...]. 
El azar lleva a grandes afinidades gráficas [...]. En esta yuxtaposición de grabados y fotografías se logran suponer las más peregrinas cosas y he visto cómo se relacionan las medusas y las bocas, así como las manos y los cactos. (id. : 645) ensuite dans l'œuvre littéraire de Ramón, tout particulièrement dans les greguerías ${ }^{13}$. Car c'est bien, je crois, le sens des greguerías que de renouveler notre perception du quotidien, en recherchant des associations d'idées inattendues et en donnant aux mots une plus grande liberté, tout en s'appuyant sur une logique poétique qui les motive, tantôt par un jeu visuel («En el papel de lija está el mapa del desierto»), un effet sonore ( El afinar los violines suena a agrio zumo de limones »), tantôt en prenant les mots au pied de la lettre : « Ballena se escribe con elle por los dos surtidores líquidos que lanza a lo alto por la nariz » (1962: 925, 379 et 193). Ce faisant, la greguería recèle une intention épistémologique ${ }^{14}$. L'auteur le rappelle dans le prologue du Total de greguerías: «La greguería, a veces, con una alusión remota y jeroglifica, con una alusión tremendamente criticable, con una pinta de un color indefinido, lo define todo. "(1962: 50-51) Il s'agit de parvenir, par les détours les moins conventionnels de la pensée, à saisir une essence des choses et à la faire connaître au lecteur. La définition semble être un prolongement naturel de la greguería, dont Ramón dit qu'elle est « el nombre más apropiado de las cosas » (id. : 72). On imagine alors sans peine l'adéquation entre l'espace du bureau ramonien, qui donne un sens nouveau aux objets qui y sont collectionnés, et la bibliothèque de travail, qui sert de creuset au programme intellectuel de la greguería.

\section{Des variations de la bibliothèque : le dictionnaire et l'inventaire}

Il reste alors un ensemble de manuscrits du fonds de Pittsburgh que je n'ai pas encore évoqués ici et qui illustrent précisément le souci épistémologique qui anime l'auteur alors même qu'il écrit, en parallèle, ses greguerías. Ramón tient en effet, comme on tient un journal, son propre dictionnaire, qu'il a baptisé Diccionario manual - en référence au dictionnaire du même nom édité par l'Académie et qui lui sert de source lorsqu'il cherche à vérifier le sens d'un mot $^{15}$. Il s'agit d'un dictionnaire revu et personnalisé, qui prend la forme d'une authentique collection de mots, germant parfois en idées de greguerías - au coin de l'une de ces fiches, on peut lire l'indication « INGREDIENTES GREGUERÍA » (Ramón Gómez de la Serna Papers: II. 37.1, fo 4). Le dictionnaire ramonien réunit, pour ce faire, différents types de travaux sur le mot: pour l'essentiel, on y trouve de longues séries de définitions, le plus souvent copiées dans le dictionnaire de la Real Academia Española qu'utilisait alors l'auteur, tantôt littéralement, tantôt retravaillées, voire réinventées par Ramón. Ainsi, sous la plume ramonienne, l'imaginero cesse de désigner un "estatuario o pintor de imágenes " (DRAE), puisqu'il faut, d'après l'auteur des Greguerías, «trasladarlo de escultor a inventor de imágenes poéticas » (II. 37. 1, fo 17a). Une part importante des fiches du dictionnaire ramonien est également occupée par des listes de mots simples, le plus souvent regroupés thématiquement. Comme une déclaration d'intention, la première fiche porte, d'ailleurs, la mention suivante: «Tenemos palabras formidables que a veces se olvidan ». Enfin, on y rencontre un certain nombre de gloses, parfois accompagnées de dessins, qui offrent les commentaires personnels de l'auteur autour de mots précis : il peut s'agir de remarques sur l'orthographe d'un mot donné, sur l'adéquation entre son signifiant et signifié ou encore de la création-justification d'un néologisme. Voici 
quelques exemples de ces prémices de greguerías: "Albor = color del alba ", "Albor alborescente ", "Arboretum = acuarium de árboles » ou encore "Carbón = luz fósil » (Ramón Gómez de la Serna Papers: II. 37). Comme les néologismes cités précédemment, on remarquera que chacune de ces définitions recherche au-delà du sens une forme de cohérence inscrite dans la chair du langage: le plus souvent, ici, par un jeu d'échos sonores (albor semble être une contraction d'alba et de color, ce qui permet à l'auteur de corriger l'acception du DRAE, «luz del alba ${ }^{16} »$, en la motivant par un effet paronymique). D'autres définitions, comme celle du charbon, reposent sur une analogie soulignée par un effet d'oxymore. Dans tous les cas - et il ne s'agit que d'un échantillon très limité-, Ramón entreprend de renommer les choses pour leur donner sens, un sens bien souvent poétique mais sémantiquement motivé. Il ne faisait, d'ailleurs, pas l'ombre d'un doute pour Joaquín de Entrambasaguas que «lo que [Ramón] ve con su mirada, ya no puede verse de otra manera; queda para la eternidad definitivamente definido y sus definiciones son más definitorias que ningunas»(1961: 1009). Aussi les définitions ramoniennes (tant les greguerías que les entrées personnalisées du Diccionario manual) participent-elles bien de la logique de la bibliothèque et des objets exposés dans le bureau, dans la mesure où elles composent une sorte de recueil encyclopédique ouvert.

Je voudrais alors, pour conclure sur la pratique de collection de mots à l'œuvre dans le « dictionnaire » ramonien, revenir sur l'une des définitions, qui en résume à mes yeux le sens : «INVENTARIO = no se recuerda muy bien porque equivoca con invención. Inventar es lo que hace el escritor al inventar o inventariar." (Ramón Gómez de la Serna Papers: II.5) L'imbrication entre les verbes "inventer" et «inventorier» est telle que le nom inventario est associé immédiatement avec inventar. On passe de la paronymie à la synonymie entre les deux verbes et c'est inventar qui conduit vers inventariar, non le contraire. L'inventaire est le produit naturel de l'acte de création; Ramón n'y voit là aucune contradiction, puisque, pour décrire, par exemple, son travail de "pescador de greguerías", il déclare : "Trato de volver a marcar todas las cosas y todos los sentimientos, con interés, y al mismo tiempo con modestia y con amor. » (1962: 31) Il s'agit d'embrasser le réel dans son ensemble - le greguerista est, avant tout, un collectionneur - et, littéralement, d'y apposer son sceau. Tout comme pour la pratique de la collection, la démarche est présentée ici comme analogue à l'acte de nommer le monde : Ramón applique son regard créateur sur le réel pour donner aux objets et aux phénomènes qui le composent un nom juste, c'est-à-dire pour arriver à en avoir une pleine connaissance. Greguería après greguer ía, il cherche, comme le dit Martínez Collado, à « llegar a un conocimiento más profundo de la realidad y a una transformación de la misma » $(1988: 10)$. En ce sens, les différentes facettes de la bibliothèque de travail - la collection de mots, de textes et d'images que s'était constituée Ramón dans son bureau - trouvent leur prolongement créatif dans la greguería à double titre : tout d'abord, parce que dans l'une et l'autre de ces démarches, il y a de la part de l'auteur une intention épistémologique, mais aussi parce que la collection - ainsi que le suggère la définition de l'inventaire-invention - est à l'origine du processus de création.

29 Au terme de cette analyse, diverses fonctions de la bibliothèque de travail de Ramón Gómez de la Serna se dégagent : une fonction créatrice (la bibliothèque en tant qu'espace d'inspiration), une fonction sociale (espace de légitimation) et une fonction de définition de l'univers mental de l'écrivain à travers une sorte d'épistémologie poétique en lien avec la pratique de la collection. Je souscris pleinement à l'affirmation de Stéphane Lojkine, selon lequel la notion de bibliothèque permet de penser «non plus en termes de texte, 
mais d'espace » (2007). La bibliothèque ramonienne est, en ce sens, un espace démultiplié en une diversité de pratiques, non pas uniquement un espace de lecture dans lequel Ramón se serait contenté d'amasser des livres. Il s'agit bien plutôt d'un espace créé comme on crée une œuvre d'art pour servir de ferment à l'œuvre littéraire de l'auteur, ainsi qu'en témoigne le processus de sélection et d'agencement des diverses collections (de citations, d'images collées aux murs et d'objets exposés) qui coexistent dans le bureau ramonien.

Or, cet espace patiemment conçu et bâti par l'écrivain-collectionneur finit par façonner à son tour son créateur. Dans un essai de 1934, «Las cosas y el ello », Ramón dépeignait le monde comme un assemblage d'objets superposés et affimait que les choses que nous côtoyons finissent par «tomber dans notre inconscient " pour le modeler à leur image (1934: 65). À l'évocation de l'espace créatif ramonien, on ne saurait imaginer Gómez de la Serna comme auteur d'une œuvre monumentale purement linéaire (qui serait plutôt l'apanage de l'érudit à la maison « con ladrillos de libros » évoqué plus haut). En revanche, l'esthétique du fragment poétique à l'œuvre dans les greguerías ou les proses brèves (articles, caprichos, courts essais ou encore épisodes juxtaposés de son Automoribundia) semble prendre toute sa place dans la bibliothèque de travail que j'ai présentée ici.

\section{BIBLIOGRAPHIE}

BAYARD Pierre (2007), Comment parler des livres que l'on n'a pas lus?, Paris : Éditions de Minuit.

BENJAMIN Walter (2015 [1931]), Je déballe ma bibliothèque. Une pratique de la collection, Paris : Payot \& Rivages.

CARDona Rodolfo (1957), Ramón: A Study of Gómez de la Serna and His Works, New York : Eliseo Torres \& Sons.

CARDona Rodolfo (1989) «Del archivo de Ramón en la Universidad de Pittsburgh: El hombre de alambre », Boletín de la Fundación Federico García Lorca, 5, 11-20.

CHARTIER Roger (2015), La main de l'auteur et l'esprit de l'imprimeur, Paris : Gallimard.

ENTRAMBASAGUAS Joaquín de (1961), « Ramón Gómez de la Serna », Las mejores novelas contemporáneas, 8, 907-1064.

GómEZ DE LA SERNA Ramón (1917), Greguerías, Valence : Prometeo.

Gómez DE LA SERNA Ramón (1999 [1924]), La sagrada cripta de Pombo (éd. de A. Trapiello), Madrid : Visor Libros.

GómEZ DE LA SERNA Ramón (1927), Greguerías escogidas, Paris, Madrid, Lisbonne : Agencia Mundial de Librería.

GómEZ DE LA SERNA Ramón (1934), « Las cosas y el ello », Revista de Occidente, 134, 190-208.

GÓMEZ DE LA SERNA Ramón (1936), « Las palabras y lo indecible », Revista de Occidente, 151, 56-85.

GóMEZ DE LA SERNA Ramón (1941), Retratos contemporáneos, Buenos Aires : Suramericana. 
GÓMEZ DE LA SERNA Ramón (1948), Automoribundia, Buenos Aires : Suramericana.

GómEZ DE LA SERNA Ramón (1952), Greguerías 1940-1952 (5éd.), Buenos Aires : Espasa-Calpe.

Gómez DE LA SERnA Ramón (1962), Total de greguerías (2éd.), Madrid : Aguilar.

HeLMich Werner (1982), « Ideología literaria y visión del mundo en las greguerías de Ramón

Gómez de la Serna », Iberoromania, 16, 54-83.

LAGET Laurie-Anne (2012), La fabrique de l'écrivain, Madrid : Casa de Velázquez.

LOJKINE Stéphane (2007), « La bibliothèque comme dispositif. La non-lecture selon P. Bayard », Acta fabula, 8(2), <www.fabula.org/revue/document2983.php> (1 ${ }^{\mathrm{er}}$ août 2015).

LOREnZo José (1930), « El día de Ramón Gómez de la Serna », ABC (Madrid, 7 décembre 1930), 3-6.

MARINAS José Miguel (2001), La fábula del bazar. Orígenes de la cultura del consumo, Madrid : Machado Libros.

MARTínez Collado Ana (1988), Ramón Gómez de la Serna: una teoría personal del arte, Madrid : Tecnos.

MELot Michel (2007), « La bibliothèque multimédia contemporaine », C. Jacob (dir.), Lieux de savoir : espaces et communautés (637-653), Paris : Albin Michel.

RAMÓN GÓMEZ dE LA SERNA PAPERS (2012), 1906-1967, SC.1967.04, Special Collections Department, University of Pittsburgh, <http://digital.library.pitt.edu/cgi-bin/f/findaid/findaid-idx? type=simple; $c=$ ascead;view=text;subview=outline;didno=US-PPiU-sc196704> $\left(1^{\mathrm{er}}\right.$ août 2015).

Sото M. (1968), « Visita a los trastos de Ramón. “Su despacho era su inspiración” (dice la esposa del escritor). "Mi marido amaba las cosas" ", Informaciones (2 janvier 1968).

\section{ANNEXES}

\section{Index onomastique des Gregues}

Cet index amende et complète celui établi par Alan Hoyle, que j'ai pu consulter à l'université de Pittsburgh. Je n'y recense les citations des auteurs apparaissant sur les coupures de presse que lorsqu'il s'agit d'auteurs par ailleurs présents sous la forme de citations manuscrites, afin de m'assurer qu'il s'agit bien d'un choix de Ramón Gómez de la Serna et non d'un hasard dû au fait que celui-ci colle parfois des pages entières d'imprimés (la plupart du temps des Selecciones del Reader's Digest) sur lesquelles figurent, pêle-mêle plusieurs dizaines de citations d'auteurs très variés.

\begin{tabular}{|l|l|}
\hline Achard, Juliette : & 1 \\
\hline Abril de V., Xavier : & 7 \\
\hline Alberti, Rafael : & 7 \\
\hline Albornoz, Álvaro de : & 30 \\
\hline Aleixandre, Vicente : & 5 \\
\hline Al-Khayyam, Omar : & 1 \\
\hline
\end{tabular}




\begin{tabular}{|c|c|}
\hline Allais, Alphonse : & 3 \\
\hline Altolaguirre, Manuel : & 1 \\
\hline Anouilh, Jean : & 4 \\
\hline Apollinaire : & 7 \\
\hline Aragon, Louis : & 4 \\
\hline Aristote : & 1 \\
\hline Arp, Hans : & 18 \\
\hline Árabes: & 3 \\
\hline Azorín: & 4 \\
\hline Bacarisse, Mauricio : & 4 \\
\hline Balzac, Honoré de : & 4 \\
\hline Barbey d'Aurevilly, Jules : & 25 \\
\hline Baudelaire, Charles: & 4 \\
\hline Beauvoir, Simone de : & 1 \\
\hline Bécquer, Gustavo Adolfo : & 4 \\
\hline Bierce, Ambrose : & 15 \\
\hline Blake, William : & 8 \\
\hline Borges, Jorge Luis : & 3 \\
\hline Bourget, Paul: & 2 \\
\hline Brazil : & 2 \\
\hline Breton, André : & 31 \\
\hline Buck, Pearl : & 1 \\
\hline Busch, Wilhelm : & 10 \\
\hline Butler, Samuel : & 2 \\
\hline Calvo, Luis: & 1 \\
\hline Campoamor, Ramón de : & 1 \\
\hline Casal, Julián del : & 3 \\
\hline
\end{tabular}




\begin{tabular}{|c|c|}
\hline Cernuda, Luis : & 19 \\
\hline Cervantes, Miguel de: & 5 \\
\hline Champion, Leo: & 2 \\
\hline Chazal, Malcolm de : & 12 \\
\hline Chesterton: & 7 \\
\hline Chino (proverbio, fábula, poema): & 10 \\
\hline Churchill, Winston: & 1 \\
\hline Ciria y Escalante, José de : & 2 \\
\hline Clarasó, Noel : & 65 \\
\hline Cocteau, Jean : & 24 \\
\hline Colette: & 2 \\
\hline Colombia: & 6 \\
\hline Confucius: & 2 \\
\hline Crane, Stephen : & 2 \\
\hline Croce, Gotardo: & 13 \\
\hline Cunningham, James Vincent: & 1 \\
\hline Dadaístas: & 5 \\
\hline Dalí, Salvador: & 2 \\
\hline D’Annunzio, Gabriele : & 1 \\
\hline Darío, Rubén : & 4 \\
\hline Dekobra, Maurice : & 5 \\
\hline Démocrite : & 3 \\
\hline Diccionario del humor francés: & 43 \\
\hline Diego, Gerardo : & 8 \\
\hline Disraeli, Benjamin : & 1 \\
\hline Doral González, Antonio : & 6 \\
\hline D'Ors, Eugenio: & 2 \\
\hline
\end{tabular}




\begin{tabular}{|c|c|}
\hline Drouet, Minou : & 11 \\
\hline Duchamp, Marcel : & 8 \\
\hline Du Fu : & 3 \\
\hline Dumas, Alexandre (fils) : & 1 \\
\hline Dunham, Katherine : & 1 \\
\hline Eça de Queiroz, José Maria : & 1 \\
\hline Eschyle : & 2 \\
\hline Éluard, Paul : & 58 \\
\hline Emerson, Ralph Waldo: & 4 \\
\hline Epictète : & 1 \\
\hline Épicure : & 1 \\
\hline Érasme : & 2 \\
\hline Ercilla y Z., Alonso de : & 5 \\
\hline Euras, Salvador : & 4 \\
\hline Faguet, Émile : & 1 \\
\hline Fargue, Léon-Paul : & 1 \\
\hline Fénelon : & 2 \\
\hline Fernández Moreno, B. : & 87 \\
\hline Fernández Moreno, Clara: & 3 \\
\hline Flaubert, Gustave : & 1 \\
\hline France, Anatole : & 1 \\
\hline Franklin, Benjamin : & 3 \\
\hline Fuertes, Gloria : & 37 \\
\hline García Lorca, Federico : & 89 \\
\hline Gautier, Théophile : & 1 \\
\hline Géraldy, Paul: & 1 \\
\hline Gide, André : & 1 \\
\hline
\end{tabular}




\begin{tabular}{|c|c|}
\hline Goethe, Johann Wolfgang von: & 2 \\
\hline Goll, Ivan : & 5 \\
\hline Gómez Carrillo, Enrique : & 1 \\
\hline Góngora, Luis de : & 9 \\
\hline González Lanuza, Eduardo : & 2 \\
\hline Gourmont, Remy de : & 2 \\
\hline Gracián, Baltazar : & 3 \\
\hline Guasti, Amerigo : & 1 \\
\hline Guillén, Jorge : & 2 \\
\hline Güiraldes, Ricardo : & 10 \\
\hline Guitry, Sacha : & 3 \\
\hline Hartmann, Paul : & 2 \\
\hline Hebbel, Friedrich : & 15 \\
\hline Heine, Heinrich : & 6 \\
\hline Hello, Ernest : & 1 \\
\hline Héraclite: & 1 \\
\hline Herford, Oliver : & 2 \\
\hline Hernández, Miguel : & 3 \\
\hline Herold, Don : & 1 \\
\hline Herrera y Reissig, Julio : & 1 \\
\hline Hesse, Hermann : & 1 \\
\hline Hitchcock, Alfred : & 1 \\
\hline Hölderlin, Friedrich : & 2 \\
\hline Holmes, Oliver Wendell : & 1 \\
\hline Hubbard, L. Ron : & 2 \\
\hline Hugo, Victor : & 18 \\
\hline Huidobro, Vicente : & 8 \\
\hline
\end{tabular}




\begin{tabular}{|c|c|}
\hline Hurtado de Mendoza, Diego : & 4 \\
\hline Huxley, Aldous : & 1 \\
\hline Jacob, Max : & 10 \\
\hline Jammes, Francis : & 1 \\
\hline Japonesas : & 12 \\
\hline Jardiel Poncela, Enrique : & 25 \\
\hline Jarry, Alfred : & 3 \\
\hline Jiménez, Juan Ramón : & 8 \\
\hline Johnson, Samuel : & 2 \\
\hline Joubert, Joseph : & 2 \\
\hline Kafka, Franz: & 2 \\
\hline Karr, Alphonse : & 1 \\
\hline Keats, John : & 2 \\
\hline La Bruyère, Jean de : & 1 \\
\hline La Fontaine, Jean de : & 2 \\
\hline La Rochefoucauld, F. de : & 2 \\
\hline Lamartine, Alphonse de : & 3 \\
\hline Lao-Tseu: & 1 \\
\hline Larrea, Juan : & 12 \\
\hline Larreta, Enrique : & 4 \\
\hline Laura : & 16 \\
\hline Lautréamont : & 3 \\
\hline Lin YuTang: & 4 \\
\hline Longanesi, Leo: & 1 \\
\hline Lope de Vega: & 3 \\
\hline Lord Byron : & 1 \\
\hline Lugones, Leopoldo: & 10 \\
\hline
\end{tabular}




\begin{tabular}{|c|c|}
\hline Machado, Antonio: & 1 \\
\hline Machado de Asis, Joaquim Maria : & 2 \\
\hline Maeztu, Ramiro de : & 1 \\
\hline Maïakowski, Wladimir : & 7 \\
\hline Mallarmé, Stéphane: & 3 \\
\hline Maritain, Jacques : & 1 \\
\hline Marc Aurèle : & 1 \\
\hline Marx, Groucho : & 2 \\
\hline Matores, Manuel : & 1 \\
\hline Maugham, William Somerset: & 1 \\
\hline Milosz, Oscar W. de L. : & 13 \\
\hline Miquelarena, Jacinto : & 14 \\
\hline Mistral, Gabriela : & 1 \\
\hline Monroe, Marilyn : & 1 \\
\hline Montesquieu : & 1 \\
\hline Morand, Paul : & 2 \\
\hline Moreto, Agustín : & 5 \\
\hline Muñoz Seca, Pedro : & 1 \\
\hline Musset, Alfred de : & 2 \\
\hline Mussolini, Benito : & 1 \\
\hline Napoléon : & 7 \\
\hline Neruda, Pablo: & 65 \\
\hline Novalis : & 4 \\
\hline O. Henry: & 1 \\
\hline Orientales: & 15 \\
\hline Ortega y Gasset, José : & 10 \\
\hline Ory, Eduardo de : & 10 \\
\hline
\end{tabular}




\begin{tabular}{|c|c|}
\hline Osler, William : & 1 \\
\hline Ovide : & 2 \\
\hline Panero, Leopoldo María : & 5 \\
\hline Paulhan, Jean : & 1 \\
\hline Péret, Benjamin : & 2 \\
\hline Picasso, Pablo: & 4 \\
\hline Pindare : & 3 \\
\hline Pitigrilli : & 2 \\
\hline Prados, Emilio : & 1 \\
\hline Primo de Rivera, José A.: & 1 \\
\hline Proust, Marcel : & 5 \\
\hline Quevedo y V., Francisco de : & 27 \\
\hline Rachilde: & 1 \\
\hline Rega Molina, Horacio : & 14 \\
\hline Renard, Jules : & 66 \\
\hline Reverdy, Pierre : & 1 \\
\hline Rey, Étienne : & 18 \\
\hline Richter, Hans : & 2 \\
\hline Rilke, Rainer Maria : & 3 \\
\hline Rimbaud, Arthur : & 33 \\
\hline Rosales, Luis : & 4 \\
\hline Rowland, Helen : & 1 \\
\hline Rueda, Salvador: & 6 \\
\hline Ruso: & 1 \\
\hline Saint Jérôme : & 1 \\
\hline Saint-Pol-Roux : & 25 \\
\hline Salinas, Pedro: & 3 \\
\hline
\end{tabular}




\begin{tabular}{|c|c|}
\hline Sand, George : & 1 \\
\hline Sappho: & 4 \\
\hline Sénèque : & 3 \\
\hline Seral y Casas, Tomás : & 29 \\
\hline Shaw, George Bernard: & 10 \\
\hline Shakespeare, William : & 5 \\
\hline Socrate: & 1 \\
\hline Sofovich, Luisita : & 1 \\
\hline Soler y Pérez, Francisco : & 39 \\
\hline Sophocle: & 1 \\
\hline Stendhal: & 2 \\
\hline Schwob, Marcel: & 28 \\
\hline Surrealista(s): & 8 \\
\hline Swinburne, Algernon: & 1 \\
\hline Tagore, Rabindranath : & 2 \\
\hline Teresa de Ávila : & 1 \\
\hline Tinayre, Marcelle : & 1 \\
\hline Torre, Guillermo de : & 1 \\
\hline Twain, Marc: & 6 \\
\hline Tzara, Tristan : & 4 \\
\hline Unamuno, Miguel de: & 3 \\
\hline Valéry, Paul : & 6 \\
\hline Virgile : & 4 \\
\hline Villiers de l'Isle Adam : & 1 \\
\hline Voltaire : & 2 \\
\hline Wilde, Oscar: & 14 \\
\hline Zamacois, Eduardo: & 3 \\
\hline
\end{tabular}


Zorilla, José : $\quad 2$

\section{NOTES}

1. Plusieurs articles parurent dans la presse espagnole et latino-américaine de façon posthume, dans les semaines qui suivirent la disparition de l'auteur.

2. L'alphabet se décline, chez Ramón, au gré des greguerías: «La A es la tienda de campaña del alfabeto ", "La B es el ama de cría del alfabeto ", « La ñ es una n con el pelo rizado » ou encore « La W es una M haciendo la plancha $(1962: 245,548,1474,632)$.

3. Ces trois (re)définitions des lettres de l'alphabet parurent dans Alpes et Pyrénées ( G, c’est le cor », «L, c'est la jambe et le pied », «Le Z, c'est l'éclair, c'est Dieu », Paris : P. Mouillot, [s. d.] : 28). On remarquera que la traduction espagnole fait ressortir la parenté avec les greguerías, en adoptant, pour les deux dernières phrases, une forme de définition de type ENTRÉE (de dictionnaire) : définition.

4. On pourrait comparer ce fragment à deux greguerías ramoniennes, certes assez dissemblables en apparence, mais qui jouent de façon analogue sur l'image de la lune: «Los gatos se beben la leche de la luna en los platos de las tejas » ou encore "Lo que le da más horror a la luna es el bostezo del cocodrilo » (Gómez de la Serna, 1962 : 414 et 419).

5. On remarquera, en effet, que sur de nombreux feuillets de Gregues, Ramón copiait indifféremment des citations allogènes et des (auto)citations de ses greguerías (clairement identifiées comme «mías»). De façon générale, des 4774 citations qui constituent la collection des Gregues, un tiers environ sont d'authentiques greguerías ramoniennes, presque toujours entremêlées avec les textes d'autres auteurs.

6. Ce qui est chronologiquement impossible, puisque le prologue du Total de greguerías est rédigé en 1955 et reproduit à l'identique dans l'édition de 1962. Or, 1955 est précisément l'année de la découverte de Minou Drouet par Françoise Sagan et de la publication de son premier recueil.

7. Pour les possibles modèles francophones, on pourra consulter le chapitre II de mon ouvrage sur la formation littéraire ramonienne, La fabrique de l'écrivain (Laget, 2012 : 127-272).

8. Si le prologue du Total de greguerías comprend une section clairement identifiée de qasidas et de haïkus, on peut se demander si Ramón préparait de nouvelles sections similaires de citations, brésiliennes ou russes par exemple, pour montrer la diffusion de la greguería.

9. Il n'est pas anodin que Luisa Sofovich ait vendu simultanément ces deux archives ramoniennes et je trouve remarquable la façon dont elle lie étroitement le sort du bureau et de la bibliothèque dans sa correspondance avec Rodolfo Cardona : «Estoy de acuerdo con todo lo que me dice y dice a los de su Universidad y espero que todo llegue a buen término, sin demorarse demasiado, porque estoy en deuda con Madrid, dilatando fechas pues allí me esperan día a día. Quisiera poder estar allí a mediados de abril, una vez que estén en viaje los canastos que llevan el "despacho" ramoniano a su sede que debe ser Madrid. » (Cardona, 1989 : 11) Aux yeux de Luisa Sofovich, la transmission du bureau et celle de la bibliothèque à deux entités publiques (musée et bibliothèque universitaire de recherche) sont les deux versants d'une « empresa de propagación y conservación del genio español que tuvimos el privilegio de conocer» (id.).

10. Le bureau le plus célèbre de Ramón, qui reçut le nom de Torreón, était la tour d'un immeuble de la rue Velázquez, à Madrid, que l'auteur occupa de 1922 à 1933.

11. Par analogie avec l'expression qui désigne la chambre noire : cámara de trabajo < cámara oscura .Si l'on file la métaphore, c'est dans cet espace qu'il développe ses images poétiques.

12. Consultables en ligne. Voir, en particulier, <http://hemeroteca.abc.es/nav/Navigate.exe/ hemeroteca/madrid/abc/1930/ 12/07/004.html> et <http://hemeroteca.abc.es/nav/ Navigate.exe/hemeroteca/madrid/abc/1930/12/07/006.html>. 
13. À défaut de bouche-méduse, voici, par exemple, une chevelure de méduse : « La cabellera de la mujer dormida es medusa del mar del sueño » (1962: 225).

14. C'est ce que constate également Werner Helmich : «El substrato decisivo de [los] juegos [verbales de la greguería] consiste en una confianza lingüistica elemental, que a pesar de todo obscurecimiento semántico surgido entretanto concede al lenguaje una función epistemológica. » (1982 : 74)

15. Une édition critique de cette somme de brouillons inédits est en cours, en collaboration avec la professeur Herlinda Charpentier Saitz de l'université du Massachusetts-Lowell. Nous y indiquerons notamment l'édition précise du Diccionario manual de la RAE que nous avons identifiée comme source de l'auteur.

16. Je cite la deuxième acception du Diccionario manual de $1927: 75$.

\section{RÉSUMÉS}

Cet article porte sur la bibliothèque de travail de l'écrivain Ramón Gómez de la Serna aujourd'hui conservée à l'université de Pittsburgh et qui renferme les derniers manuscrits et matériaux de recherche de l'auteur. Cette bibliothèque est ici envisagée comme une matérialisation de la «bibliothèque intérieure » ramonienne, offrant à l'auteur une diversité de fonctions (créatrice et sociale, notamment) et nous donnant une vision d'ensemble de son univers esthétique.

El presente artículo reflexiona sobre la biblioteca de trabajo de Ramón Gómez de la Serna hoy conservada en la Universidad de Pittsburgh, que contiene los últimos manuscritos y materiales de investigación del autor. La analizaré aquí como una plasmación de la «biblioteca interior» ramoniana en sus distintas funciones, en especial las creativas y personales, para ver en qué medida nos ayuda a entender de manera privilegiada y global el universo estético de Ramón Gómez de la Serna.

This paper is about the research library of the spanish writer Ramón Gómez de la Serna kept as a Special Collection at the Hillman Library (Pittsburgh). It includes Ramón's last manuscripts and research documents. I consider it as an expression of the Ramonian "inner library" in its different functions (especially, the creative and social ones), that allows us to have a comprehensive view of this writer's aesthetic world.

\section{INDEX}

Mots-clés : bibliothèque de travail, espace créatif, pratique de la collection, université de Pittsburgh

Palabras claves : Biblioteca de trabajo, espacio creativo, coleccionismo, Universidad de Pittsburgh

Keywords : research library, creative Space, collection, Pittsburgh University 


\section{AUTEUR}

\section{LAURIE-ANNE LAGET}

Université Paris-Sorbonne - Paris 4, CRIMIC 